of the random cloud model suggest a plausible alternative picture of interstellar space. According to the proposed hypothesis the major part of the gaseous medium forms an extensive, unhomogeneous cloud partaking of the galactic rotation. It is this diffuse gas which produces a line in every star and the strongest component of a multiple line. The weaker components with a large velocity dispersion arise from currents resembling large-scale turbulent elements in the diffuse cloud. The line widths expected from this model appear to be consistent with the observations.

A similar irregular diffuse distribution is suggested for the interstellar grains, although the evidence indicates substantial differences in the behavior of the density fluctuations for the two forms of matter. The proposed general distribution of the interstellar particles receives support from studies in bright regions of the Milky Way.

Wayne University, Detroit, Mich.

Ewen, H. I. and E. M. Purcell. Radiation from hyperfine levels of interstellar hydrogen.

The hyperfine transition in the ground state of atomic hydrogen with frequency I420.405 Mc/ sec has been detected in galactic radiation. ${ }^{1}$ The hydrogen is apparently an extended source concentrated in the plane of the local galaxy. Measurements were made at declination $-5^{\circ}$ with a calculated antenna beam width of approximately $\mathrm{I} 2^{\circ}$ between half-power points. The line as measured in the vicinity of the galactic center appears in emission with temperature difference $40^{\circ} \pm 5^{\circ} \mathrm{C}$ with respect to the radiation field and with a width of approximately $80 \mathrm{kc}$. The line shows a Doppler shift which is in reasonable agreement with the earth's orbital motion and the motion of the solar system. Evidence that the source is extended is provided by the variation of Doppler shift during the time of observation.

The apparatus, consisting essentially of a superheterodyne receiver and large horn-type antenna, differs from a conventional radiometer by comparing the radiation temperature of the line with the background temperature in the same spectral region.

The line was first detected on March 25, I95I. The results were immediately communicated to the radio astronomy groups in Holland under the direction of Dr. J. H. Oort and in Australia under the direction of Dr. J. L. Pawsey. Both have completed construction of the necessary equipment and a recent communication from Holland confirms the discovery.

This research was partly supported by a grant from the Rumford Fund, American Academy of Arts and Sciences.

I. A. G. Prodell and P. Kusch, Phys. Rev, 79, I009, Department of Physics, Harvard University, Cambridge, Mass.

Gaposchkin, Sergei. The system of $R Z$ Eridani and a new bright eclipsing variable with double-lined spectrum.

\section{RZ ERIDANI}

The eclipsing variable RZ Eridani has been investigated on $2 \mathrm{I} 84$ photographic plates of the Harvard patrol collection and on the basis of $5^{2}$ radial velocities published by Cesco and Sahade. The elements are:

$$
\text { Min. = JD 2429982.37 + 39.28248IE. }
$$

The maximum brightness is 8.04 mag., the primary minimum is I.70 mag. deep and the secondary is only 0.04 mag. deep. The duration of the primary eclipse is $\mathrm{O}^{\mathrm{P}} \mathrm{O} .5$, one of the shortest among the eclipsing systems. The duration of the "totality" is about $\mathrm{o}^{\mathrm{P}}$.or 8 . The photometric orbit is eccentric and in rare conformity with the spectroscopic one. The light of the bright component, eclipsed at the primary minimum, is 0.79 , its relative radius is 0.04 in units of the distance between the centers of the components, and in solar units it is about 2.0; its absolute magnitude is about $+2 . \mathrm{I}$. It is too bright and too large for its spectrum. The fainter component is probably a subgiant with rather peculiar properties in size and luminosity which are in definite disagreement with the subgiant class. Photometrically it should be one spectral class later, $M$ instead of G8.

Since the brighter component is a "metallic" star, we have an indication that a metallic star is brighter than an ordinary main sequence star for the same spectral class.

There is definite additional variation of brightness during the phase of totality, about o.Io mag., which is real and comes from the fainter component which is 3.33 times larger than the brighter one. The inclination of the photometric orbit is $87^{\circ} \cdot 5$. All values are given for the "uniform" solution. The "darkened" data are also 